\title{
Mechanically-induced proximal arterial occlusion and stent- within-a-stent technique for the treatment of bilateral vertebral artery dissecting aneurysms
}

\author{
${ }^{1} \mathrm{~T}-\mathrm{K}$ KIM, MD, ${ }^{1} \mathrm{~S}$ I SEO, MD, ${ }^{1} \mathrm{H}$ Y SEOL, MD, ${ }^{2} \mathrm{~B}$ J KWON, MD and ${ }^{2} \mathrm{M}$ H HAN, MD \\ ${ }^{1}$ Department of Diagnostic Radiology, Korea University, College of Medicine, Seoul and \\ ${ }^{2}$ Department of Radiology, Seoul National University College of Medicine, Seoul, Korea
}

\begin{abstract}
Several endovascular approaches have been used in the treatment of dissecting aneurysms of the vertebrobasilar system. We present a case of a bilateral spontaneous vertebral artery (VA) dissecting aneurysms presenting with a subarachnoid haemorrhage. The dominant left VA aneurysm was treated with a stentwithin-a-stent construct and the contralateral VA aneurysm was proximally occluded, with no complications, by an unintentional mechanically-induced arterial occlusion.
\end{abstract}

\author{
Received 12 July 2005 \\ Revised 9 January 2006 \\ Accepted 13 January 2006 \\ DOI: $10.1259 /$ bjr/89133751 \\ (C) 2006 The British Institute of \\ Radiology
}

Dissecting aneurysms of the vertebral artery (VA) are uncommon, but constitute a significant cause of nontraumatic subarachnoid haemorrhage (SAH). Reported rates for recurrent haemorrhage from a dissecting VA aneurysm range between $30 \%$ and $70 \%$, with a high mortality rate estimated at $46 \%$. Due to the frequent incidence of re-bleeding, in most reported cases patients have undergone either surgical or endovascular therapy $[1,2]$.

A variety of endovascular treatment strategies have been applied to the treatment of VA dissecting aneurysms. These techniques have included trapping of the affected segment, proximal occlusion, stent-assisted coil embolisation and stent-assisted angioplasty [3-7]. The most complete treatment of a dissecting aneurysm would be to exclude the segment with a trapping procedure. Although coil occlusion of the affected segment is one strategy, it poses the risk of occlusion of side branches, further injury to the vessel wall, or dislodging emboli [3]. Proximal occlusion can be considered in cases in which the dissected segment is deemed to be too risky to cross, or too fusiform or narrow to accept coils, or in cases in which the dissected segment incorporates important branches, including the anterior inferior cerebellar artery, the posterior inferior cerebellar artery (PICA), or the anterior spinal artery [3, 4]. Stent-assisted angioplasty is another therapeutic option for dissecting aneurysms. With the advent of navigable intracranial stents, VA aneurysms are now amenable to endovascular stent placement. The stent can be used to exclude the aneurysm from the circulation and preserve the parent artery [5-7]. Here, we report the endovascular treatment of a case of bilateral VA

Address correspondence to: Taik-Kun Kim, MD, Department of Diagnostic Radiology, Korea University College of Medicine, Ansan Hospital, \#516 Kojan-Dong, Ansan City, Kyungki-Do 425-020, Korea. E-mail: tkkim@kumc.or.kr. dissecting aneurysms, in which the dominant VA was reconstructed via a stent-within-a-stent technique and the contralateral VA, which exhibited severe narrowing below the PICA with a pseudoaneurysm, was proximally occluded, with no complications, by an unintentional mechanically-induced arterial occlusion.

\section{Clinical presentation}

A 45-year-old man presented with an acute-onset severe headache, nausea and an episode of syncope. The patient's haemodynamic and respiratory parameters remained stable. Upon admission, the patient appeared alert and orientated, but suffered from a persistent headache. Upon examination, he exhibited nuchal rigidity. We discovered no focal cranial nerve or motor deficits. CT on admission showed no evidence of SAH. Repeated lumbar punctures yielded many old-form red blood cells. We confirmed acute SAH. Clinically, Hunt-Hess grade was I. Angiography of the right VA showed severe narrowing below the PICA, with a focal pseudoaneurysm in the middle of the narrowed segment. Angiography of the dominant left VA revealed a dissecting aneurysm, exhibiting fusiform dilatation, as well as a visible intimal flap in the infra-PICA location (Figure 1).

As there was poor collateral flow through both sides of the posterior communicating artery, the preservation of arterial continuity was inevitable, at least from one VA. Therefore, we proposed endovascular reconstruction of the dominant left VA and occlusion of the right VA.

\section{Intervention}

With the patient under sedation, we positioned a $6 \mathrm{~F}$ guiding catheter (Guider Softip; Target Therapeutics, Fremont, CA) at the origin of the dominant left VA. We 


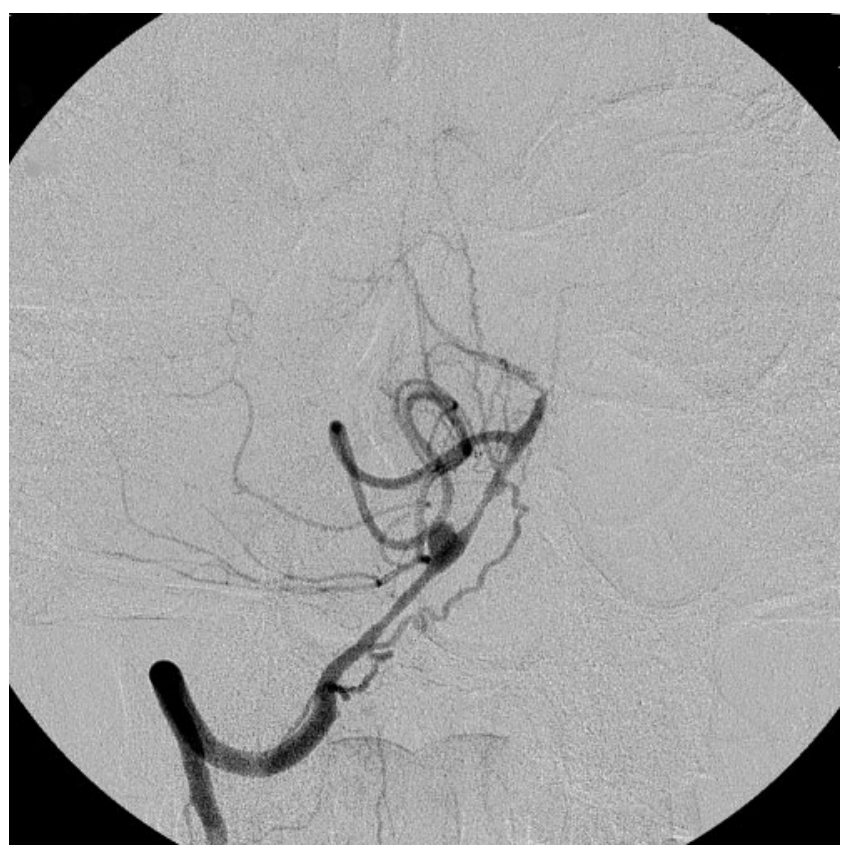

(a)

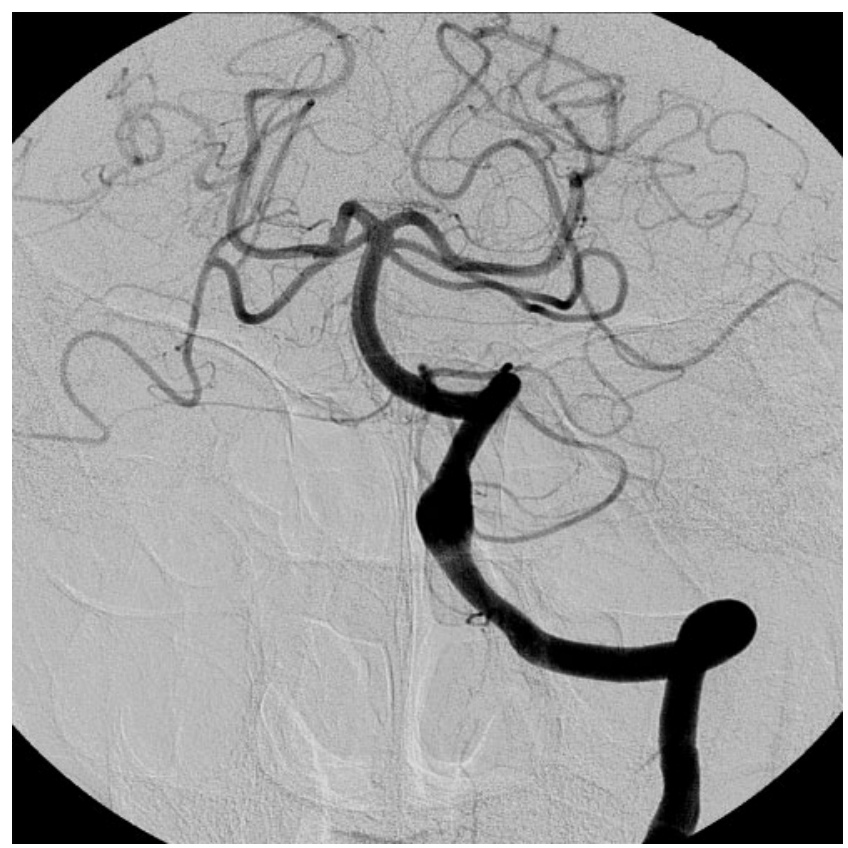

(b)

Figure 1. Initial angiography. (a) Right vertebral artery (VA) injection angiography shows the eccentric location of the aneurysm relative to the V4 segment of the VA. Tapered proximal and distal narrowing suggests dissection. (b) Left VA injection angiography reveals a fusiform aneurysm in the infra-PICA location.

then performed selective angiography, and the targeted segment was outlined in multiple projections with rotational angiography. We detected a fusiform dilatation with an intimal flap in the infra-PICA location. The aneurysmal segment was then crossed under road mapping with a microcatheter (Renegade; Target) over a 0.014 inch wire (Transcend; Target). After repeated rotational angiography for the confirmation of the catheter's position, we advanced a 0.014 inch, $300 \mathrm{~cm}$ long exchange microguidewire (Choice PT; Boston Scientific, Miami, FL) across the lesion site, ultimately positioning it at the distal basilar artery. The microcatheter was then exchanged for a stent-delivery catheter. A $3.5 \mathrm{~mm} \times 28 \mathrm{~mm}$ balloon-mounted coronary stent (Vision; Guidant, Santa Clara, CA) was advanced over the microguidewire and positioned at the location to cover the dilated aneurysmal sac. The balloon was inflated to the maximum recommended pressure in order to deploy and implant the stent into the inner arterial wall. Then, $3.5 \mathrm{~mm} \times 23 \mathrm{~mm}$ and $3.0 \mathrm{~mm} \times 18 \mathrm{~mm}$ balloon-mounted stents (Vision; Guidant) were implanted sequentially within the first stent. Angiography after the procedure resulted in good stent placement and patency. Angiography revealed residual opacification, but we also noted a significant reduction in the amount of flow into residual sac (Figure 2a,b).

The guiding catheter was then repositioned at the origin of the right VA, under roadmapping, over an angled 0.035 inch Terumo standard angiography guidewire, which was positioned up to the C3 level. After withdrawing the guidewire, a test injection through the guiding catheter into the right VA revealed complete occlusion of the right VA at the C5 level, which coincided with a site which had been winding in an unusual fashion on a previous angiography (Figure 2c,d). A bolus of $2 \mathrm{mg}$ of verapamil was administered through a guiding catheter, but was of no avail. After withdrawing the guiding catheter to the aortic arch, we waited, eventually performing an evaluation of the patient's neurological status. The patient was found to exhibit neither symptoms nor signs of right VA occlusion. After $30 \mathrm{~min}$, fluorography revealed a contrast column in the proximal right VA, without distal movement (Figure 2e). Angiography of the left VA demonstrated the reversal of the flow from the vertebrobasilar junction to the right PICA and to the dissecting aneurysm (Figure 2f). As the flow to the dissected segment was found to be weak and slow, we decided to treat the dissecting aneurysm conservatively. The following day, left VA angiography revealed a further reduction in the retrograde flow to the right infra-PICA dissection, with preservation of the flow in the right PICA. Again, right subclavian artery angiography evidenced complete occlusion, with further thrombosis occurring downward from the C5 level. The patient continued to be neurologically intact, and was discharged after 8 days. Follow-up angiography of the patient's right innominate artery after 8 months revealed complete occlusion of his right VA, with a minute stump filling (Figure 3a). Left VA angiography revealed the absence of flow below the right PICA, while flow to the right PICA was preserved, and also revealed a decreased fusiform aneurysm in the left infra-PICA location, with patency consistent with the normal calibre of the vessel (Figure 3b).

\section{Discussion}

There are currently a variety of available endovascular treatment strategies for VA dissecting aneurysms. As in our case, the endovascular management of VA is 


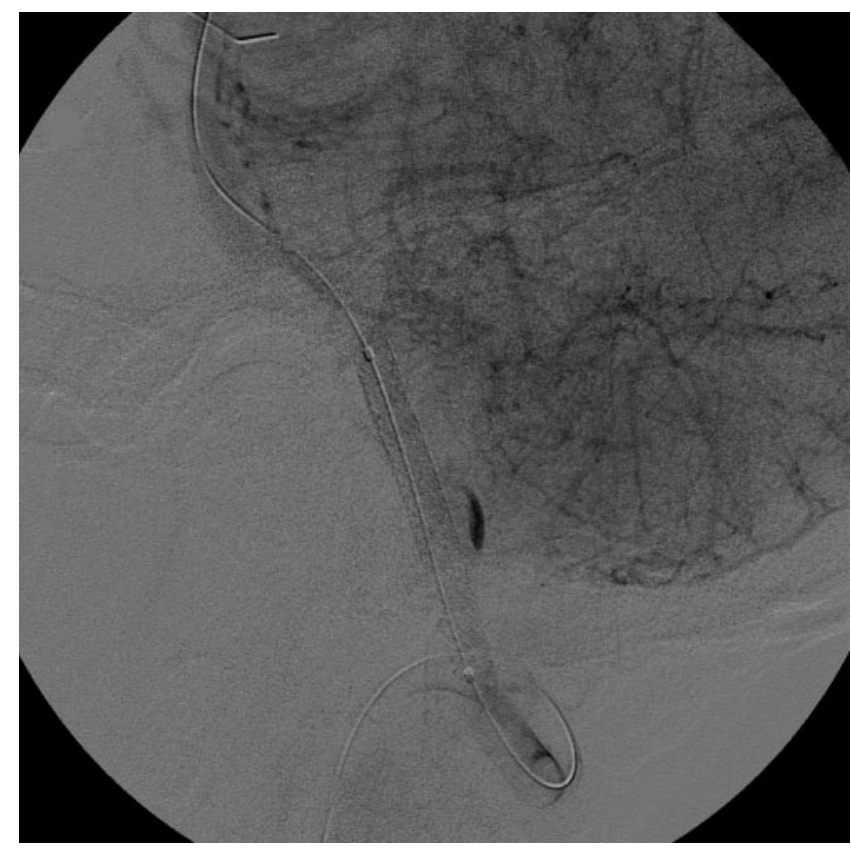

(a)

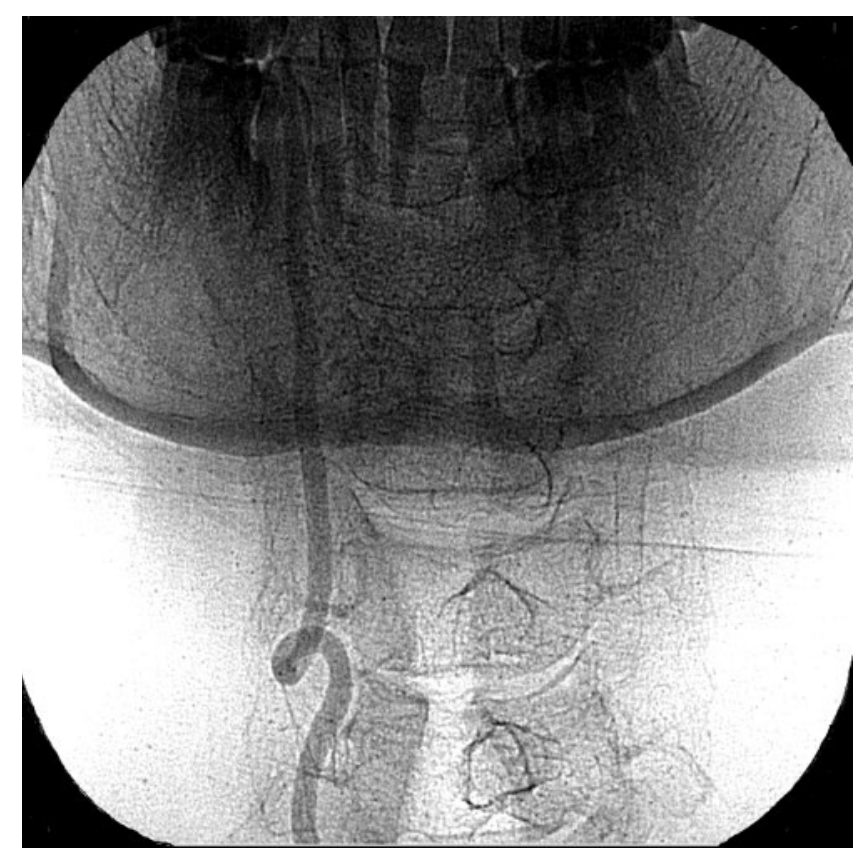

(c)

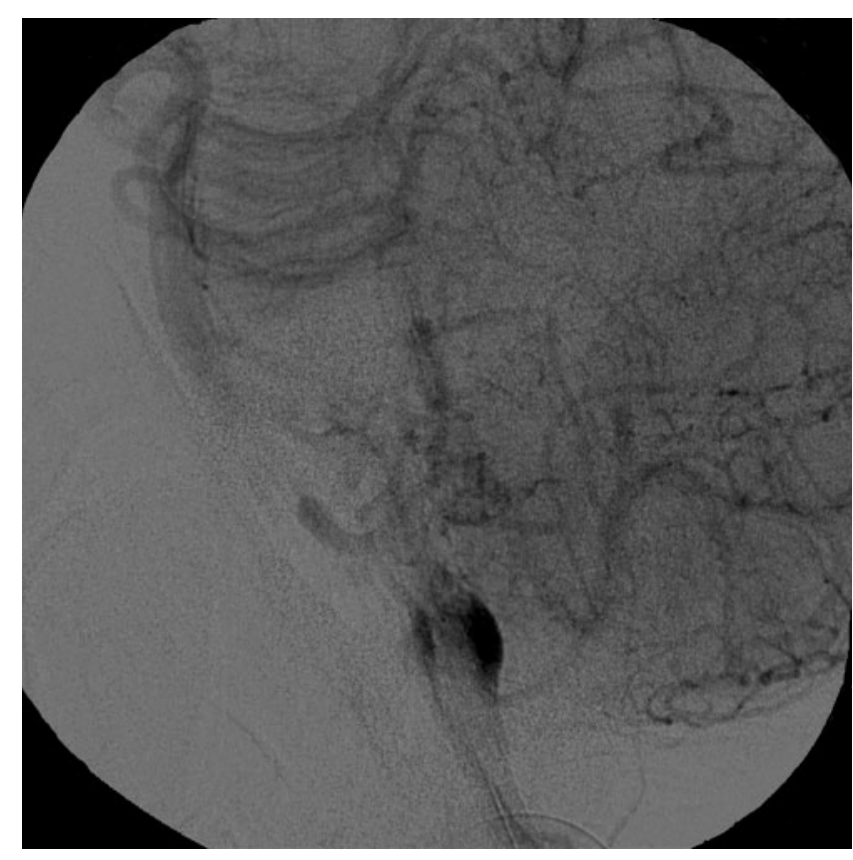

(b)

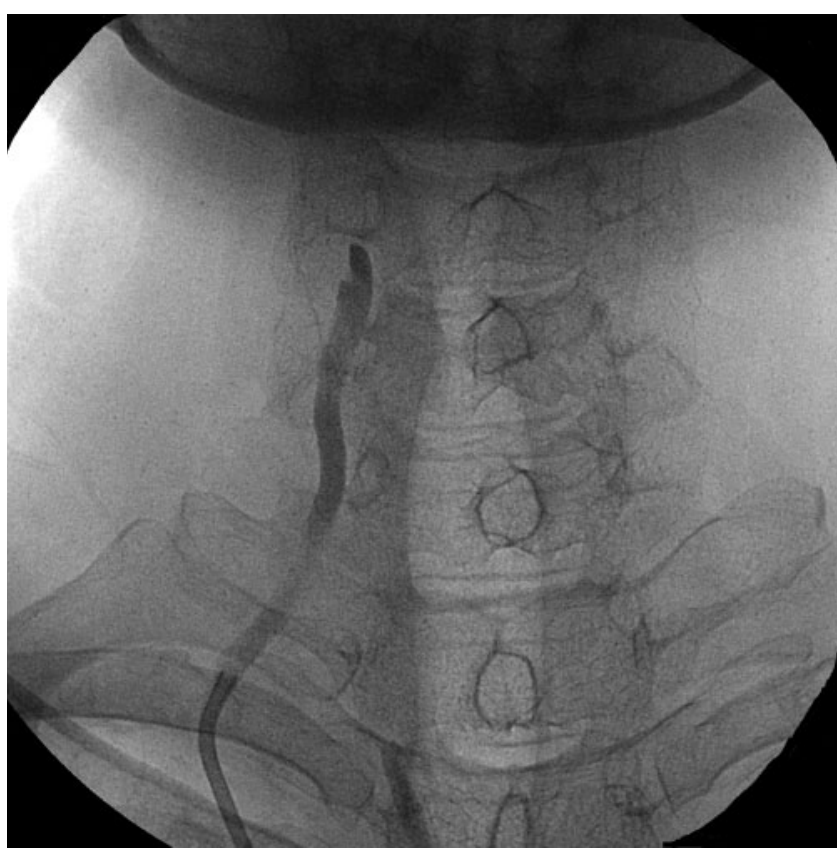

(d)

Figure 2. Intervention. (a) Lateral left vertebral artery (VA) angiography after the implantation of one coronary stent reveals a partial contrast stasis in the aneurysmal sac in the venous phase. (b) Lateral left VA angiography after three coronary stents implanted with a "stent-within-a-stent" technique reveals further stasis in the aneurysmal sac in the venous phase. Stents are barely visible due to their poor radiopacity. (c) Right VA test injection before the positioning of the guiding catheter reveals an unusually winding course at C5 level. (d) Right VA test injection immediately after the positioning of the $6 \mathrm{~F}$ guiding catheter at the origin of the right VA reveals complete occlusion at the C5 level. (Continued)

dependent on the manner in which the condition presents. In cases involving bilateral VAs similar to ours, a diverse combination of therapeutic options can be applied. In addition, the preservation of arterial continuity in at least one VA can sometimes be much more important than the immediate exclusion and thrombosis of the aneurysm.

The advent of navigable intracranial stents has allowed these stents to be used to exclude the VA aneurysm from the circulation, thereby preserving the parent artery $[7,8]$. Some authors have advocated the use of a stent as a buttress for the implantation of detachable coils or similar embolic agents [8]. More recently, the stent-within-a-stent technique has been used for the treatment of dissections in the cardiology field, and even in some cases for intracranial dissection [9, 10]. This simple modification of an established endovascular approach attempts to create a pseudocovered stent to 


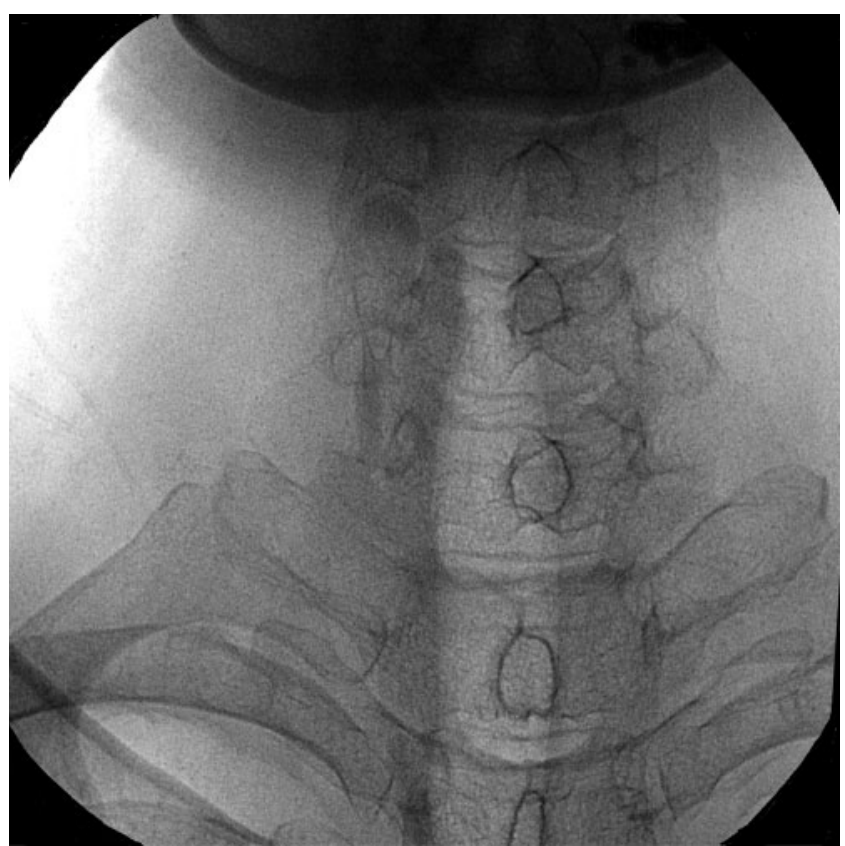

(e)

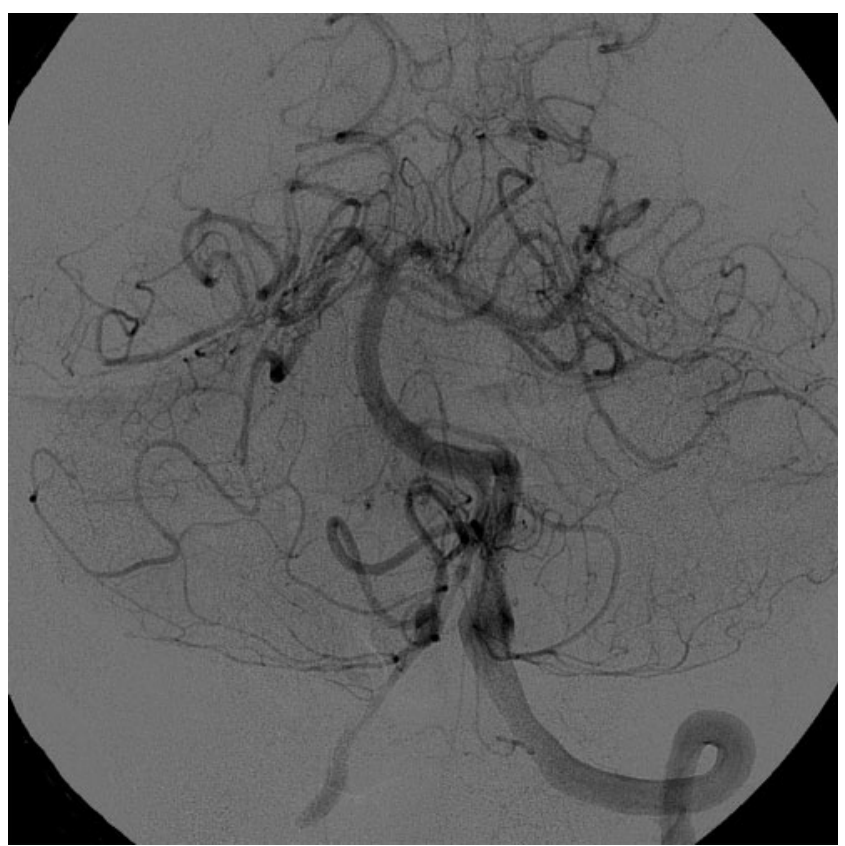

(f)

Figure 2. (Cont.) (e) Fluorography of the neck $30 \mathrm{~min}$ after the right VA occlusion reveals contrast stasis without any movement, although a bolus of verapamil was administered and the guiding catheter had been withdrawn. (f) Left VA angiography after the right VA occlusion reveals the retrograde filling of the right PICA and infra-PICA dissecting aneurysm.

exclude the aneurysm from its parent vessel, and to preserve arterial continuity. In the reported cases, immediate or delayed exclusion and thrombosis of the aneurysm was accomplished with no significant complications, at follow-up durations ranging between 6 months and 17 months [10]. In the case presented here, the patient's dominant left VA was treated via a "stent-within-a-stent" technique, employing three coronary stents. With an increase in the number of deployed stents, our angiography revealed slowing of the filling of the aneurysmal sac and further stasis. Although enthusiasm for endovascular stent placement techniques in the treatment of cerebrovascular disease is increasing, this method has several obvious limitations in the treatment

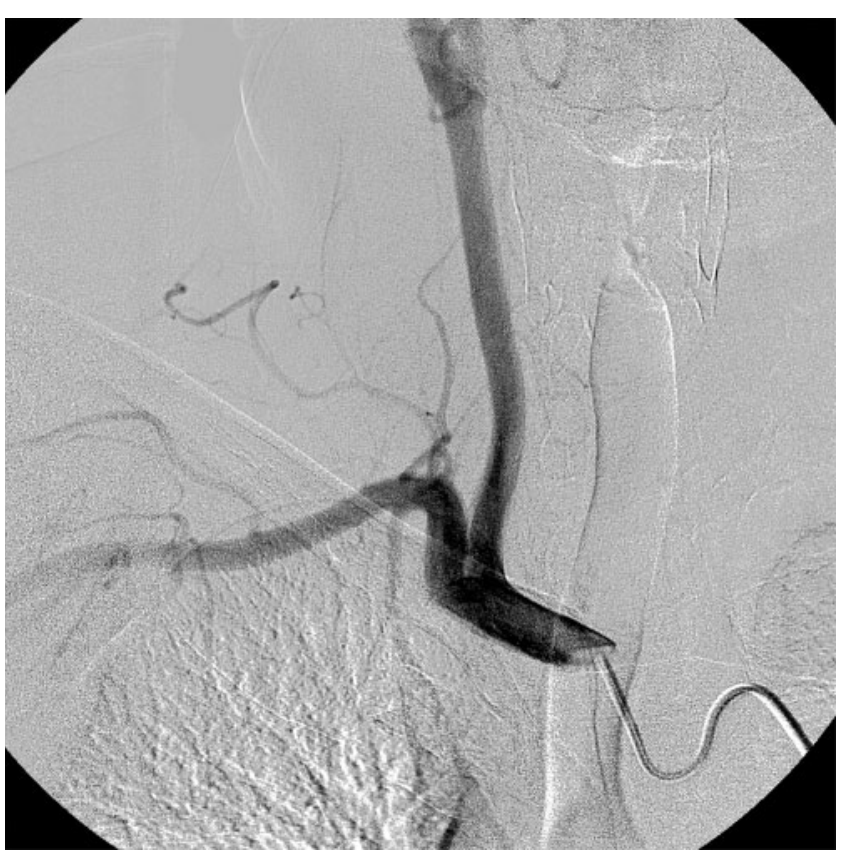

(a)

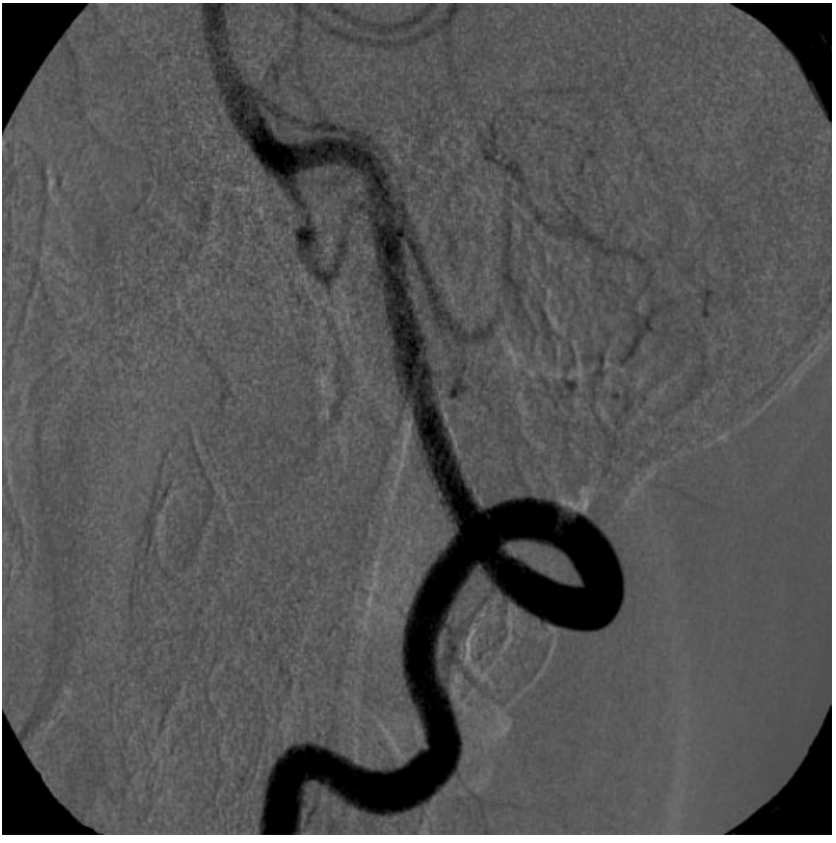

(b)

Figure 3. 8 months after. (a) Right innominate artery angiography revealed complete occlusion of the right vertebral artery (VA) with a minute stump filling. (b) Lateral left VA angiography revealed absent flow below the right PICA, with a preservation of flow to the right PICA and a decreased fusiform aneurysm of the left infra-PICA location, with patency of the stents. 
of all aneurysms [10]. Most intracranial aneurysms occur at vessel bifurcations and in close association with small perforating branches. Placement of a stent across these bifurcations and the ostia of small perforating branches risks vessel occlusion. In addition, catheter manipulation and stent placement in the distal vertebral and vertebrobasilar junction risks further dissection, occlusion, or perforation. The patency rate of stents in the intracranial circulation is unknown, but it most likely decreases over time. The risk of acute stent thrombosis increases, particularly as stent diameters decrease to less than $4 \mathrm{~mm}$. Stent porosity or the ratio of metal to tissue is another factor associated with neointimal growth. As the porosity decreases or as the stent surface area increases, the risk of subacute thrombosis from excessive neointimal growth increases. Covered stents can be chosen for treating dissecting aneurysm as an alternative device, but higher-profile devices are more difficult to navigate around the tortuous intracranial circulation. In addition, self-expandable stents would be a more ideal treatment option than balloon-expandable stents in "stent-withina-stent" technique as self-expandable stents have better adaptability to the tortuous vessels and have excellent radial force without recoiling. However, higher-profile and foreshortening would be still challenging issues for intracranial usage. The current limitations will inevitably be mitigated by improvements in stent technology. As stent technology evolves, so too will its applications. Although this case was one with a limited follow-up period, the complete exclusion of the aneurysmal sac upon follow-up angiography led us to believe that our strategy constitutes a feasible technique for the dissecting aneurysm of dominant VA.

Proximal occlusion of the parent artery from which the aneurysm arises has become a widely accepted approach to the treatment of vertebral dissecting aneurysm without manipulation of the affected segment, in cases in which more direct endovascular occlusions or stent placements cannot be safely performed. As recent studies have shown $[3,4,6]$, proximal occlusion is a feasible therapeutic option, especially in cases in which complete thrombosis can be achieved upon immediate or delayed angiography. In our case, occlusion of the right VA at the most proximal level of the dissecting aneurysm was initially proposed. However, arterial occlusion developed immediately after the guiding catheter was positioned at the origin of the right VA. The level of the occlusion coincided with an unusually winding course in the right VA, through which only an angled standard angiography guidewire had been placed throughout the procedure. It is not clear whether the occlusion at C5 level is related to direct arterial damage by the guiding wire itself, although the catheter had never crossed the occluded level, or if the occlusion is related to a severe arterial spasm of the diseased vessel. However, as shown in this case, once the proximal occlusion was achieved there was no longer any need to manipulate the artery to resolve the arterial occlusion. Because there were no significant side branches from the beginning of the right VA and the aneurysmal segment, occlusion level made no differences when complete proximal occlusion was achieved. Re-evaluating this case, we found that the verapamil administration had no effect and did not constitute a necessary measure. On the contrary, restoration of blood flow could be hazardous because of embolus dislodgement or haemorrhage from the dissecting aneurysm. Thus, we suggest that proximal occlusion with coils at the more proximal level than the previously occluded site would have been a more reasonable strategy in order to prepare against spontaneous recanalization after a certain period. To our knowledge, this report represents the first reported instance of mechanically-induced arterial occlusion in the treatment of a VA dissecting aneurysm, although this was an unintended outcome of our procedures. This report presents a case with a limited follow-up period; however, this case illustrates that an inadvertently occluded parent artery can consequently generate positive results in the treatment of VA dissecting aneurysm.

\section{References}

1. Yamaura A, Watanabe $Y$, Saeki N. Dissecting aneurysms of the intracranial vertebral artery. J Neurosurg 1990;72:183-8.

2. Mizutani T, Aruga T, Kirino T, Miki Y, Saito I, Tsuchida T. Recurrent subarachnoid hemorrhage from untreated ruptured vertebrobasilar dissecting aneurysms. Neurosurgery 1995;36:905-13.

3. Rabinov JD, Hellinger FR, Morris PP, Ogilvy CS, Putman $\mathrm{CM}$. Endovascular management of vertebrobasilar dissecting aneurysms. AJNR Am J Neuroradiol 2003;24:1421-8.

4. Leibowitz R, Do HM, Marcellus ML, Chang SD, Steinberg GK, Marks MP. Parent vessel occlusion for vertebrobasilar fusiform and dissecting aneurysms. AJNR Am J Neuroradiol 2003;24:902-7.

5. Willing SJ, Skidmore F, Donaldson J, Nobo UL, Chernukha $\mathrm{K}$. Treatment of acute intracranial vertebrobasilar dissection with angioplasty and stent placement: report of two cases. AJNR Am J Neuroradiol 2003;24:985-9.

6. Cohen JE, Gomori JM, Umansky F. Endovascular management of spontaneous bilateral symptomatic vertebral artery dissections. AJNR Am J Neuroradiol 2003;24:2052-6.

7. Sekhon LH, Morgan MK, Sorby W, Grinnell V. Combined endovascular stent implantation and endosaccular coil placement for the treatment of a wide-necked vertebral artery aneurysm: technical case report. Neurosurgery 1998;43:380-4.

8. Lanzino G, Wakhloo AK, Fessler RD, Hartney ML, Guterman LR, Hopkins LN. Efficacy and current limitations of intravascular stents for intracranial internal carotid, vertebral, and basilar artery aneurysms. J Neurosurg 1999;91:538-46.

9. Hamon M, Monassier JP. Stenting within a stent for treatment of residual dissection. Cathet Cardiovasc Diagn 1997;40:319-21.

10. Mehta B, Burke T, Kole M, Bydon A, Seyfried D, Malik G. Stent-within-a-stent technique for the treatment of dissecting vertebral artery aneurysms. AJNR Am J Neuroradiol 2003;24:1814-8. 